Constraints on brane and bulk ideal fluid in Randall-Sundrum cosmologies

\author{
Enqvist, Kari
}

2001-07-27

Enqvist , K , Keski-Vakkuri , E \& Räsänen , S 2001, ' Constraints on brane and bulk ideal

fluid in Randall-Sundrum cosmologies ' , Physical Review D , vol. 64 , no. 4 . https://doi.org/10.1103/PhysRevD.64.0

http://hdl.handle.net/10138/310206

https://doi.org/10.1103/PhysRevD.64.044017

other

acceptedVersion

Downloaded from Helda, University of Helsinki institutional repository.

This is an electronic reprint of the original article.

This reprint may differ from the original in pagination and typographic detail.

Please cite the original version. 
hep-th/0007254

HIP-2000-39/TH

December 18, 2000

\title{
Constraints on Brane and Bulk Ideal Fluid in Randall-Sundrum Cosmologies
}

\author{
Kari Enqvist地, Esko Keski-Vakkurið and Syksy Räsänen円 \\ ${ }^{1,2}$ Helsinki Institute of Physics \\ P.O. Box 9, FIN-00014 University of Helsinki, Finland \\ and \\ ${ }^{1,3}$ Department of Physics \\ P.O. Box 9, FIN-00014 University of Helsinki, Finland
}

\begin{abstract}
We investigate constraints for including bulk and brane matter in the RandallSundrum model. In static configurations with two zero thickness branes, we find that no realistic brane matter is possible. We also consider the possibility that the radion has stabilized by dissipating its energy into the bulk in the form of some unspecified matter, and find the Randall-Sundrum cosmological solutions in the presence of bulk ideal fluid. We discover that the metric is necessarily in a static configuration. We also discover that there is only one allowed equation of state for the bulk fluid, $p=\rho$, corresponding to the stiff ideal fluid. We find the corresponding brane cosmologies and compare them with the Friedmann-Robertson-Walker model.
\end{abstract}

\footnotetext{
${ }^{1}$ E-mail: kari.enqvist@helsinki.fi

${ }^{2}$ E-mail: esko.keski-vakkuri@hip.fi

${ }^{3}$ E-mail: syksy.rasanen@helsinki.fi
} 


\section{Introduction}

Randall and Sundrum have recently suggested a novel solution to the hierarchy problem involving extra dimensions 11, 2], which has attracted much attention. The RS proposal assumes a five-dimensional spacetime where the extra dimension is a $S^{1} / Z_{2}$ orbifold, with branes located at the two orbifold fixed points. The branes are thus at the spatial boundaries of the bulk spacetime. The brane tensions together with a negative bulk vacuum energy provide the source for the 5-d Einstein equation, which yields as the solution the metric

$$
d s^{2}=e^{-2 k|y|}\left(-d t^{2}+\sum_{i=1}^{3}\left(d x^{i}\right)^{2}\right)+d y^{2} .
$$

The exponential warp factor rescales the physical masses on the second "TeV brane", giving rise to a large suppression factor with respect to the Planck mass, and hence a possible solution to the hierarchy problem. However, this is achieved at the expense of fine tuning: the cosmological constants on the two branes must be of equal magnitude but with opposite signs. Also, the size of the extra dimension, or the radion, is a four dimensional modulus field whose value needs to be fixed at the right scale in order for the RS scenario to work.

The RS model has been refined in many ways since its inception, but the question of radion stabilization remains unsolved. According to commonly accepted wisdom, moving branes would give rise to particle masses which change in time [3], so that the radion should be very nearly stabilized by the beginning of nucleosynthesis so as not to conflict with observation. One possibility was proposed by Goldberger and Wise, who assumed the existence of a massive bulk scalar field with self-interactions on the branes [4]; see also [5, 6]. By integrating over the fifth dimension one then generates an effective potential for the radion, which has a non-trivial minimum. As the radion field settles into the minimum, the size of the extra dimension gets fixed.

Using a bulk scalar field to stabilize the radion has also been studied in [7]. Other ideas for fixing the size of the fifth dimension include gaugino condensation in a supersymmetric setting [8] and the possibility that the Hubble redshift might damp the radion so that it is almost stabilized [9] A phenomenological mechanism for stabilizing the radion was considered in [3].

The implications of radion stabilization independent of the specifics of the stabilization mechanism were considered in [11, 12]. It was found that a fixed size for the fifth dimension requires a certain non-constant form for the $y y$-component of the bulk energy-momentum

\footnotetext{
${ }^{4}$ In $[10$ the interesting possibility that an asymptotically constant radion emerges from the Einstein equation naturally without the introduction of extra degrees of freedom was considered. Unfortunately the Ansatz used actually precludes any dynamics for the size of the fifth dimension.
} 
tensor. This form turned out to be determined by a constraint that related the $y y$ component of the stress tensor to its trace. This constraint could be understood in terms of the backreaction of the radion to the inclusion of matter.

Whatever the actual mechanism for stabilizing the radion, it is natural to assume that the fixed size of the extra dimension results from dynamical evolution. In the GoldbergerWise scenario, the radion would evolve from its initial state towards the minimum by dissipating the extra energy away. The possibility of the radion stabilizing via decay into Standard Model particles on the $\mathrm{TeV}$ brane was considered in [3]. However, it does not seem farfetched to assume that there are other fields in the bulk, which can couple to the radion, whether directly, via the GW field or via some other mechanism, providing an alternative channel for energy dissipation. Such additional fields could e.g. be a part of the field content of a supergravity theory in the bulk. The asymptotic final state in the bulk would then involve the radion field with a constant value dictated by the minimum of the effective potential, together with some bulk matter.

If bulk matter exists, at large times one would expect it to be in the state of maximum entropy. This means that e.g. viscous flow should eventually get damped away, and that the bulk matter can be considered an ideal fluid. In this case, stress tensor of the bulk fluid will be spatially isotropic, in particular the $y y$-component will not differ from the other diagonal spatial components, unlike in previous investigations [7, 10, 11, 12, 13, 14, 15, 16].

In section 2 we find the general form of the metric assuming homogeneity and isotropy with respect to the three visible spatial dimensions, one static extra dimension and no matter flow along this extra dimension. We then focus on two-brane systems with zero thickness branes, and make a distinction between static and non-static configurations. We investigate constraints on brane matter in two-brane systems, and show that in static configurations the only allowed forms of brane matter are a cosmological constant and domain walls moving at the speed of light. Single-brane configurations easily allow generic matter, but we are interested in the possibility of adding another brane necessary for generating the hierarchy. We then comment on the scenarios for including realistic brane matter in non-static two-brane configurations. In section 3 we consider the case of an ideal fluid plus a cosmological constant in the bulk and find the exact cosmological solutions. Curiously, we find that the metric must be in a static configuration, leaving no room for realistic brane matter, and that there is only one allowed equation of state for the bulk fluid, $p=\rho$, corresponding to the stiff ideal fluid. We note that it is possible for both branes to have a positive cosmological constant, and one brane may even have a zero cosmological constant, a result discovered in [17] and emphasized in [7]. In section 4 we discuss our results. 


\section{Warped spacetimes bounded by two branes}

\subsection{General form of the metric}

The object of our interest is the Einstein equation in $4+1$ dimensions,

$$
G_{A B}=\kappa^{2} T_{A B}
$$

In the above, $\kappa^{2}=1 / M^{3}$, where $M$ is the Planck scale in five dimensions. The indices $A$ and $B$ run through time $t$, three spatial coordinates $x^{i}$ with infinite range and a compact spatial coordinate $y$. We use the standard notational convention and denote partial derivatives with respect to $t$ and $y$ by dots and primes, respectively.

We begin with two general assumptions regarding the metric and the matter content:

1. The spacetime is homogeneous and isotropic with respect to the three spatial coordinates $x^{i}$.

2. There is no matter flow in the $y$-direction.

Assumption 1. The most general metric obeying homogeneity and isotropy with respect to the spatial coordinates $x^{i}$ is

$$
d s^{2}=-n(t, y)^{2} d t^{2}+2 c(t, y) d t d y+b(t, y)^{2} d y^{2}+\frac{a(t, y)^{2}}{\left(1+\frac{K}{4} r^{2}\right)^{2}} \sum_{i=1}^{3}\left(d x^{i}\right)^{2}
$$

where $r^{2}=\sum_{i=1}^{3}\left(x^{i}\right)^{2}$ and $6 K$ is the constant three-dimensional spatial curvature. While the general form of the metric includes a $d t d y$ cross term, most of the literature has adopted Gaussian normal coordinates, where the cross term is absent $(c=0)$. The same assumption will be adopted in the present paper. We call the metric with $c=0$ the cosmological Randall-Sundrum metric in contrast to the original static Randall-Sundrum solution. For simplicity of notation, we put $K=0$ for the rest of this work. This does not affect our essential results.

The Einstein Equation. The nontrivial components of the Einstein equation for the metric (3) in Gaussian normal coordinates, with $K=0$, are

$$
\begin{aligned}
& G_{t t}=3\left[-\frac{n^{2}}{b^{2}}\left(\frac{a^{\prime \prime}}{a}+\frac{a^{\prime}}{a}\left(\frac{a^{\prime}}{a}-\frac{b^{\prime}}{b}\right)\right)+\frac{\dot{a}}{a}\left(\frac{\dot{a}}{a}+\frac{\dot{b}}{b}\right)\right]=-\kappa^{2} n^{2} T_{t}^{t} \\
& G_{i i}=\frac{a^{2}}{b^{2}}\left[2 \frac{a^{\prime \prime}}{a}+\frac{n^{\prime \prime}}{n}+\frac{a^{\prime}}{a}\left(\frac{a^{\prime}}{a}+2 \frac{n^{\prime}}{n}\right)-\frac{b^{\prime}}{b}\left(\frac{n^{\prime}}{n}+2 \frac{a^{\prime}}{a}\right)\right]
\end{aligned}
$$




$$
\begin{aligned}
& -\frac{a^{2}}{n^{2}}\left[2 \frac{\ddot{a}}{a}+\frac{\ddot{b}}{b}+\frac{\dot{a}}{a}\left(\frac{\dot{a}}{a}-2 \frac{\dot{n}}{n}\right)+\frac{\dot{b}}{b}\left(2 \frac{\dot{a}}{a}-\frac{\dot{n}}{n}\right)\right]=\kappa^{2} a^{2} T^{i}{ }_{i} \\
G_{y y}= & 3\left[\frac{a^{\prime}}{a}\left(\frac{a^{\prime}}{a}+\frac{n^{\prime}}{n}\right)-\frac{b^{2}}{n^{2}}\left(\frac{\ddot{a}}{a}+\frac{\dot{a}}{a}\left(\frac{\dot{a}}{a}-\frac{\dot{n}}{n}\right)\right)\right]=\kappa^{2} b^{2} T^{y}{ }_{y} \\
G_{t y}= & 3\left[\frac{n^{\prime}}{n} \frac{\dot{a}}{a}+\frac{a^{\prime}}{a} \frac{\dot{b}}{b}-\frac{\dot{a}^{\prime}}{a}\right]=-\kappa^{2} n^{2} T_{y}^{t} .
\end{aligned}
$$

We are interested in models with two parallel branes at the endpoints of the $y$ coordinate. In particular, we demand that the proper transverse distance between the branes is independent of time. So we make one more assumption:

3. In the coordinates where the branes are at $y=y_{1}, y_{2}$, we have $\dot{b}=0$.

Assumptions 2 and 3. No matter flow in the $y$-direction means that $T_{t y}=0$. Inserting this into the relevant component of the Einstein equation (四), along with $\dot{b}=0$, we obtain the result

$$
\dot{a}(t, y)=A(t) n(t, y),
$$

where $A$ is an unknown function. We are interested in cosmological solutions, and so will throughout the paper assume that $\dot{a} \neq 0$.

\subsection{Motivation for a static fifth dimension}

The assumption of a static fifth dimension appears in most papers on the RS model. Since it does not seem obvious that a non-static fifth dimension would in general lead to conflict with observation, we present a brief discussion to motivate the assumption $\dot{b}=0$.

The RS model was first envisaged as a solution to the hierarchy problem: the (fourdimensional) length scale increases as one moves from one brane to the other, which induces a change in the mass scales between the branes. The hierarchy between the Planck scale on the (hidden) brane at $y=y_{1}$ and the $\mathrm{TeV}$ scale on the (observable) brane at $y=y_{2}$ requires that the functions $n(t, y)$ and $a(t, y)$ satisfy

$$
\begin{aligned}
& \frac{n\left(t, y_{1}\right)}{n\left(t, y_{2}\right)}=N \\
& \frac{a\left(t, y_{1}\right)}{a\left(t, y_{2}\right)}=N h(t),
\end{aligned}
$$

where $N \sim 10^{16}$ is the ratio of the Planck and $\mathrm{TeV}$ scales and $h(t)$ is some function of time (to allow for the possibility of different cosmological expansion factors on the two branes). This is a rather weak constraint, since it makes no reference to behaviour away 
from the branes. Therefore, in the literature there often appears a stronger and hence more pragmatic constraint. One makes the additional Ansatz that the condition (6) holds not only for the particular value $y=y_{1}$, but for all values of $y$, with the value $N$ replaced by some function $f(y)$. In other words, the Ansatz says that the Einstein equation implies no preferred brane positions. This Ansatz implies that

$$
\begin{aligned}
n(t, y) & =n_{0}(t) f(y) \\
a(t, y) & =a_{0}(t) f(y) \\
b(t, y) & =b(t, y) .
\end{aligned}
$$

Substituting (7) into the ty-component of the Einstein equation (4) and assuming $T_{t y}=0$, we obtain the condition

$$
f^{\prime} \dot{b}=0 \text {. }
$$

For a nontrivial warp factor, the above equation can only be satisfied if $\dot{b}=0$. One can then redefine the coordinates $t$ and $y$ to set the functions $n_{0}(t)$ and $b(y)$ to unity, so that the metric reads

$$
\begin{aligned}
n(t, y) & =f(y) \\
a(t, y) & =a_{0}(t) f(y) \\
b(t, y) & =1 .
\end{aligned}
$$

As an aside, we note that the redefinition of the $y$-coordinate to obtain $b=1$ contains a possible problem: if the original function $b(y)$ has zeros, the required coordinate transformation may be singular. In particular, this singularity may map either or both of the boundaries of the $y$-coordinate from finite values to infinity, making the fifth dimension non-compact. This issue was discussed in [18], and singularities in the RS scenario have more generally been considered in [17, 19, 20]. In the present work, we simply assume that the metric is non-singular so that the fifth dimension remains compact in such transformations.

We see that the factorized metric (9) and the condition $\dot{b}=0$ can be motivated by requiring Randall-Sundrum-type solutions. If there is a weak time-dependence in $b$, we might expect to approximately recover the factorizable metric (9), as noted in [21. It is known that the metric (9) does not allow for brane matter (other than the cosmological constant) [15, 21]. Next, we discuss the constraints for adding brane matter.

\subsection{Constraints on brane matter}

In addition to presenting a mechanism to generate the hierarchy, the RS model had another remarkable feature: the localization of gravity into the vicinity of the Planck 
brane. Clearly, one would like to preserve both of these features while adding matter onto the $\mathrm{TeV}$ brane. Are there then any constraints on possible equations of state for brane matter, and what features of the model are responsible for them? We will next focus on isolating the potential sources for trouble and find out what features the model should have in order to accommodate generic brane matter.

The localization of gravity is easiest to achieve in the orbifold geometry where the branes have a zero thickness? This assumption will be adopted in the present paper:

4. Both branes have zero thickness.

Assumption 4. According to the above assumption, the stress tensor of brane matter is proportional to a delta function. Thus the Einstein tensor must contain delta functions as well. The orbifolding of the $y$-coordinate introduces discontinuities in the first $y$ derivatives of the metric, and delta functions in the second $y$-derivatives. Since the metric is assumed to be continuous, the only possible delta function contributions to the Einstein tensor come from these second $y$-derivatives, $a^{\prime \prime}$ and $n^{\prime \prime}$. This severely restricts the input of the metric to the brane stress tensor (or vice versa).

The brane part of the Einstein equation (14) reads

$$
\begin{aligned}
\left.3 \frac{1}{b^{2}} \frac{a^{\prime \prime}}{a}\right|_{\delta} & =\left.\kappa^{2} T_{t}^{t}\right|_{\text {brane }} \\
\left.\frac{1}{b^{2}}\left(2 \frac{a^{\prime \prime}}{a}+\frac{n^{\prime \prime}}{n}\right)\right|_{\delta} & =\left.\kappa^{2} T_{i}^{i}\right|_{\text {brane }} .
\end{aligned}
$$

The notations $\delta$ and brane refer to the delta function parts of the derivatives and the stress tensor, respectively. With the assumption of spatial homogeneity and isotropy the general form of the brane stress tensor is

$$
\left.T_{B}^{A}\right|_{\text {brane }}=\sum_{m=1,2} \frac{\delta\left(y-y_{m}\right)}{b\left(t, y_{m}\right)} \operatorname{diag}\left(-\rho_{m}(t), p_{m}(t), p_{m}(t), p_{m}(t), 0\right)
$$

where the index $m$ enumerates the branes, and we take $y_{2}>y_{1}$. On the other hand, the delta function part of the metric is related to the jumps of the first derivatives of the metric, which can in turn be expressed in terms of the continuous part of the metric [22]:

$$
\begin{aligned}
\left.a^{\prime \prime}\right|_{\delta} & =\sum_{m=1,2} \delta\left(y-y_{m}\right)\left[a^{\prime}\right] \\
& =\sum_{m=1,2} \delta\left(y-y_{m}\right)(-1)^{m+1} 2 a_{c}^{\prime},
\end{aligned}
$$

\footnotetext{
${ }^{5}$ Branes with finite thickness have been considered in $11,12,19$.
} 
where $\left[a^{\prime}\right]$ is the discontinuity of $a^{\prime},\left[a^{\prime}(y)\right]:=\lim _{\varepsilon \rightarrow 0}\left(a^{\prime}(y+\varepsilon)-a^{\prime}(y-\varepsilon)\right)$, and $a_{c}^{\prime}$ is the continuous part of $a^{\prime}$. An identical relation holds for $n$. Putting together (10), (11) and (12), we have

$$
\begin{aligned}
& \pm\left.\frac{2}{b} \frac{a_{c}^{\prime}}{a}\right|_{y=y_{m}}=-\frac{\kappa^{2}}{3} \rho_{m} \\
& \pm\left.\frac{2}{b} \frac{n_{c}^{\prime}}{n}\right|_{y=y_{m}}=\frac{\kappa^{2}}{3}\left(3 p_{m}+2 \rho_{m}\right) .
\end{aligned}
$$

The static configuration of branes. In the simplest scenario to generate the hierarchy between the branes, one assumed that the warp factor $n$ is independent of the cosmic time $t$, so that $n=f(y)$. In fact, there is always a coordinate system where this is true. Any two-dimensional Riemannian manifold is conformally flat, so we can change to conformal coordinates in the $(t, y)$-subspace. In conformal coordinates, the metric (3) reads

$$
d s^{2}=-\tilde{b}(\tilde{t}, \tilde{y})^{2} d \tilde{t}^{2}+\tilde{b}(\tilde{t}, \tilde{y})^{2} d \tilde{y}^{2}+\tilde{a}(\tilde{t}, \tilde{y})^{2} \sum_{i=1}^{3}\left(d x^{i}\right)^{2} .
$$

We then orbifold in the $\tilde{y}$-direction, so that we have two parallel branes at the fixed points at $\tilde{y}_{1}, \tilde{y}_{2}$. Introducing the assumption $d \tilde{b} / d \tilde{t}=0$, the metric reduces to

$$
d s^{2}=-\tilde{b}(\tilde{y})^{2} d \tilde{t}^{2}+\tilde{b}(\tilde{y})^{2} d \tilde{y}^{2}+\tilde{a}(\tilde{t}, \tilde{y})^{2} \sum_{i=1}^{3}\left(d x^{i}\right)^{2} .
$$

We redefine the $\tilde{y}$-coordinate so as to render the metric into the form

$$
d s^{2}=-f(\tilde{y})^{2} d \tilde{t}^{2}+d \tilde{y}^{2}+a(\tilde{t}, \tilde{y})^{2} \sum_{i=1}^{3}\left(d x^{i}\right)^{2} .
$$

We call the above bulk-brane configuration the static configuration, since the metric components in the $(\tilde{t}, \tilde{y})$-subspace are time-independent, and the branes are not moving. Note it was crucial to introduce conformal coordinates before orbifolding. Had we orbifolded first and moved to conformal coordinates afterwards, the metric would still have the form (17), but the branes would move through the bulk instead of staying at fixed positions.

What are the constraints for brane matter in the static configuration? If $a(\tilde{t}, \tilde{y})=$ $a_{0}(\tilde{t}) f(\tilde{y})$, the only possible equation of state is that of a cosmological constant, $p_{m}=-\rho_{m}$. Giving up factorizability of $a(\tilde{t}, \tilde{y})$ does not allow much more freedom. In that case (13) allows $\rho_{m}$ to be time-dependent, but because the l.h.s of (14) is time-independent, the time-dependent part of $3 p_{m}+2 \rho_{m}$ must cancel. That corresponds to a time-dependent 
density of two-dimensional domain walls moving at the speed of light on the brane. So the most general equation of state is

$$
\rho_{m}(\tilde{t})=\rho_{m(\text { wall })}(\tilde{t})+\rho_{m(\text { vacuum })}=-\frac{3}{2} p_{m(\text { wall })}(\tilde{t})-p_{m(\text { vacuum })} .
$$

In Section 3 we will discuss constraints for bulk matter. Interestingly, we will find that the presence of an ideal fluid in the bulk will force the bulk metric to be that of a static configuration.

Several RS-type solutions containing brane matter with arbitrary equations of state have been presented [3, 11, 12, 13, 15, 21, 22, 23, 24], to mention a few. First of all, it is an easier task to find realistic brane cosmologies in a single-brane configuration. Then one is not addressing the hierarchy problem, and consequently there is more freedom in setting up the model. For example, the brane could be moving across the bulk geometry [25, 26, 27], or one can take the $n(t, y)$ factor in the metric to be in a non-factorized form. For two-brane systems, inclusion of generic matter requires non-static configurations of branes.

We will next briefly discuss the non-static configurations with two branes.

Non-static configurations. The strong constraints for the brane matter followed because we introduced conformal coordinates prior to orbifolding. Let us now see what happens if we orbifold in general Gaussian normal coordinates. We start again from the general form of the metric (3), with $c=0$ and $K=0$. We then move to the orbifold geometry, with parallel branes at the fixed points $y_{1}<y_{2}$. Further, we again require that $\dot{b}=0$ and set $b=1$. So, we are dealing with the following class of metrics

$$
d s^{2}=-n(t, y)^{2} d t^{2}+d y^{2}+a(t, y)^{2} \sum_{i=1}^{3}\left(d x^{i}\right)^{2}
$$

with a compact y-direction $y_{1} \leq y \leq y_{2}$.

It is known that if $n(t, y)$ takes a non-factorized form, there are single-brane solutions allowing generic matter.

The reference [13 presented a two-brane configuration with generic matter on the $\mathrm{TeV}$ brane, while satisfying the condition (6) up to leading order. However, the fifth dimension was required to be static only up to leading order, at higher orders the $b$-coefficient in the metric may contain time-dependent terms.

Time-dependence of $\mathbf{b}$. We discussed above that if one assumes $b$ to be fixed, whether to avoid inducing time-dependence in particle masses or for some other reason, and have found that one easily ends up with overly constrained brane matter. 
Perhaps the most viable option for including brane matter is to allow for a timedependent $b$. It has been argued that time dependence in $b$ leads to time-varying particle masses [3]. Then any change in $b$ must be negligible by the time of nucleosynthesis so as not to contradict observation. As an aside, we note that these arguments have depended on a specific form of the metric, and that it is not obvious that a time-dependent $b$ would in general lead to such time-dependence of $n$ and $a$ as to affect particle masses.

We should thus consider the possibility that $b$ is almost stabilized but varies in time slowly enough not to conflict with observation, either asymptotically approaching a constant value or oscillating about a minimum. A $b$ varying slowly should allow the inclusion of realistic brane matter. The time-independent $b$ can then be considered an approximation, and perhaps an asymptotic limit, of this scenario. Treating time-dependent brane matter as a perturbation against a background of bulk matter (including possibly a cosmological constant) and brane cosmological constants is supported by the fact that energy density of the universe from the time of nucleosynthesis onwards is quite small in natural units, even if the scale of the five-dimensional gravitation is in the TeV range. Also, it seems in general more plausible that dynamical evolution would lead to a $b$ with weak time-dependence rather than a completely fixed $b$.

We now move on to discuss constraints for bulk matter. Note that we will continue to take $b$ to be constant, and assume that a weak time-dependence (along with brane matter) could be included as a perturbation. This line of thought has been pursued in [3, 28].

\section{The Einstein Equation with Ideal Fluid}

\subsection{The stress tensor}

After general investigations of the metric and brane matter, we now proceed to study the particular case of a bulk with ideal fluid and a cosmological constant.

Bulk matter, either in the form of scalar fields or an ideal fluid (which can sometimes be interpreted as a scalar field, and vice versa) has been considered in many papers. However, the scalar field studies, of which we mention only a few, have concentrated on fixing the size of the fifth dimension [4, 5, 6], inflation [18], the cosmological constant problem [20], singularities and the AdS/CFT-correspondence [19], or on more general aspects of the formalism [6], not on obtaining cosmological solutions. In papers of a more cosmological nature, often only the $y y$-component of the bulk stress tensor has been allowed to deviate from a cosmological constant [7, 11, 12, 13, 14, and in any case the $y y$-component has been taken to be different from the $x^{i} x^{i}$-components [10, 10, 16] , so that the bulk matter

\footnotetext{
${ }^{6}[16]$ is particularly interesting in that the $y y$ - and $x^{i} x^{i}$-components are initially assumed to be different
} 
cannot be interpreted as an ideal fluid. Furthermore, complete and explicit cosmological solutions for the case of a bulk stress tensor with non-trivial $t t$ - and $x^{i} x^{i}$-components are rarely presented; 28] mentions one in passing. We will now present one such solution.

We take the branes and the bulk to contain some ideal fluids of unspecified nature. For the moment, we do not make any assumptions about the metric. It is clearest to introduce a local orthonormal frame to find the form of the stress tensor. We introduce coordinates $\left(\hat{x}^{\hat{A}}\right)=\left(\hat{t}, \hat{x}^{\hat{i}}, \hat{y}\right)$ such that locally the five-dimensional line element takes the form of the 5-d Minkowski metric,

$$
d s^{2}=-d \hat{t}^{2}+\sum_{\hat{i}=1}^{3}\left(d \hat{x}^{\hat{i}}\right)^{2}+d \hat{y}^{2}
$$

In the local orthonormal frame, the stress tensor for brane (bulk) ideal fluids must be homogeneous and isotropic in the three (four) spatial dimensions on the branes (in the bulk). We thus have

$$
\begin{aligned}
T_{\hat{B}}^{\hat{A}}= & \sum_{m=1,2} \delta\left(\hat{y}-\hat{y}_{m}\right) \operatorname{diag}\left(-\rho_{m}, p_{m}, p_{m}, p_{m}, 0\right) \\
& +\operatorname{diag}(-\rho-\Lambda, p-\Lambda, p-\Lambda, p-\Lambda, p-\Lambda)
\end{aligned}
$$

In the above, $\rho_{m}, p_{m}$ are the energy densities and pressures of ideal fluid on the two branes located at

$$
\hat{y}_{1}=\int_{0}^{y_{1}} d y^{\prime} b\left(t, y^{\prime}\right) ; \hat{y}_{2}=\int_{0}^{y_{2}} d y^{\prime} b\left(t, y^{\prime}\right)
$$

$\rho, p$ are the energy density and pressure of the bulk ideal fluid and $\Lambda$ is the bulk cosmological constant which we have for convenience separated out. In particular, note that in the local orthonormal frame the pressure of an ideal fluid in the $\hat{y}$ direction is equal to the pressure in the $\hat{x}^{\hat{i}}$ directions. We now introduce the assumption of homogeneity and isotropy in the directions parallel to the brane. Then the pressures and energy densities cannot depend on the coordinates $\hat{x}^{\hat{i}}$, only on the time $\hat{t}$ and, in the case of the bulk fluid, the perpendicular direction $\hat{y}$. Thus, expressing the stress tensor in general coordinates $x^{A}$, we have

$$
\begin{aligned}
T_{B}^{A}= & \sum_{m=1,2} \frac{\delta\left(y-y_{m}\right)}{b\left(t, y_{m}\right)} \operatorname{diag}\left(-\rho_{m}(t), p_{m}(t), p_{m}(t), p_{m}(t), 0\right) \\
& +\operatorname{diag}(-\rho(t, y)-\Lambda, p(t, y)-\Lambda, p(t, y)-\Lambda, p(t, y)-\Lambda, p(t, y)-\Lambda) .
\end{aligned}
$$

The bulk ideal fluid is assumed to satisfy a linear equation of state,

$$
p(t, y)=w \rho(t, y) .
$$

but are forced to be equal by the Ansatz used. 
We could for generality write the bulk ideal fluid as a sum of components with different $w$, but since it turns out that $w$ can take only one value, we prefer not to clutter the notation. Also, one could in principle allow the coefficient $w$ to depend on $t$ and $y$, describing a time- and coordinate-dependent (that is, interacting) mixture of ideal fluids. However, in this paper we shall assume that $w$ is constant.

\subsection{Bulk ideal fluid implies a static configuration}

We again start from the metric (3), with $c=0$ and $K=0$. Before tackling the Einstein equation, we simplify the problem by taking advantage of the conservation law of the stress tensor,

$$
D_{A} T^{A}{ }_{B}=0 \text {, }
$$

which implies that matter on the branes and in the bulk satisfies the following equations:

$$
\begin{aligned}
\dot{\rho}_{m}+\left.3\left(\rho_{m}+p_{m}\right) \frac{\dot{a}}{a}\right|_{y=y_{m}} & =0 \\
\dot{\rho}+(\rho+p)\left(3 \frac{\dot{a}}{a}+\frac{\dot{b}}{b}\right) & =0 \\
p^{\prime}+(\rho+p) \frac{n^{\prime}}{n} & =0
\end{aligned}
$$

Interestingly, (28) implies that it is not possible to have bulk ideal fluid with $p=0$ (assuming that the warp factor is non-trivial). So, the conservation law of the stress tensor already restricts the possible equations of state for the bulk ideal fluid. We will shortly see that the full Einstein equation (with the assumption $\dot{b}=0$ ) permits only one particular equation of state (aside from the equation of state of a cosmological constant), that of the stiff ideal fluid.

Substituting the equation of state (24), we can integrate the equations (27) and (28) for the bulk fluid to obtain (for $w \neq 0$ )

$$
\begin{aligned}
& \rho(t, y)=\rho_{0} B(t)^{\frac{1+w}{w}} n(t, y)^{-\frac{1+w}{w}} \\
& \rho(t, y)=\rho_{0} C(y)^{-\frac{1+w}{w}} a(t, y)^{-3(1+w)} b(t, y)^{-(1+w)}
\end{aligned}
$$

where $\rho_{0}$ is a constant and $B$ and $C$ are some unknown functions, with the powers of $w$ introduced for convenience. Excepting the case of a cosmological constant, $w=-1$, we can rewrite the equations (29) as (we introduce the assumption $\dot{b}=0$ )

$$
\begin{aligned}
& \rho(t, y)=\rho_{0} B(t)^{\frac{1+w}{w}} n(t, y)^{-\frac{1+w}{w}} \\
& n(t, y)=B(t) C(y) a(t, y)^{3 w} .
\end{aligned}
$$


The second equation in (29) is a relation between the metric functions $n$ and $a$. On the other hand, the ty-component of the Einstein equation also gives a relation between $n$ and $a$. According to (5), the assumption $T_{t y}=0$ implies (with $\dot{b}=0$ )

$$
n(t, y)=A(t)^{-1} \dot{a}(t, y)
$$

Combining the conservation law relations (30) and the no-flow relation (31), we have

$$
\begin{aligned}
\rho(t, y) & =\rho_{0} B(t)^{\frac{1+w}{w}} n(t, y)^{-\frac{1+w}{w}} \\
n(t, y) & =B(t) C(y) a(t, y)^{3 w} \\
\dot{a}(t, y) & =A(t) B(t) C(y) a(t, y)^{3 w} .
\end{aligned}
$$

We can integrate the equation for $a$ in (32) and obtain $n, a$ and $\rho$ in terms of two unknown functions of $t$ and two unknown functions of $y$. The form of these functions can be found in the Appendix.

Let us now consider the Einstein equation (四) in the bulk, with $\dot{b}=0$ (and $b=1$ ). Since the Einstein equation is local and the metric is assumed to be continuous, the branes contribute only to boundary conditions. Hence we can ignore the brane contribution to the stress tensor in local bulk calculations. With the relation (31), the ty-component of the Einstein equation is satisfied trivially. The remaining components of (4) with the stress tensor (23) and the equation of state (24) read, away from the branes:

$$
\begin{aligned}
\frac{a^{\prime \prime}}{a}+\frac{a^{\prime 2}}{a^{2}}-\frac{1}{n^{2}} \frac{\dot{a}^{2}}{a^{2}} & =-\hat{\rho}-\hat{\Lambda} \\
2 \frac{a^{\prime \prime}}{a}+\frac{n^{\prime \prime}}{n}+\frac{a^{\prime}}{a}\left(\frac{a^{\prime}}{a}+2 \frac{n^{\prime}}{n}\right)-\frac{1}{n^{2}}\left[2 \frac{\ddot{a}}{a}+\frac{\dot{a}}{a}\left(\frac{\dot{a}}{a}-2 \frac{\dot{n}}{n}\right)\right] & =3(w \hat{\rho}-\hat{\Lambda}) \\
\frac{a^{\prime}}{a}\left(\frac{a^{\prime}}{a}+\frac{n^{\prime}}{n}\right)-\frac{1}{n^{2}}\left[\frac{\ddot{a}}{a}+\frac{\dot{a}}{a}\left(\frac{\dot{a}}{a}-\frac{\dot{n}}{n}\right)\right] & =w \hat{\rho}-\hat{\Lambda}
\end{aligned}
$$

where

$$
\hat{\rho}:=\frac{\kappa^{2}}{3} \rho, \hat{\Lambda}:=\frac{\kappa^{2}}{3} \Lambda
$$

Taking linear combinations of the above equations, we obtain the following equivalent set:

$$
\begin{aligned}
-\frac{a^{\prime \prime}}{a}+\frac{n^{\prime \prime}}{n}-2 \frac{a^{\prime}}{a}\left(\frac{a^{\prime}}{a}-\frac{n^{\prime}}{n}\right)+\frac{2}{n^{2}}\left(-\frac{\ddot{a}}{a}+\frac{\dot{a}}{a}\left(\frac{\dot{a}}{a}+\frac{\dot{n}}{n}\right)\right) & =3(w+1) \hat{\rho} \\
2 \frac{a^{\prime \prime}}{a}+\frac{n^{\prime \prime}}{n} & =(w-1) \hat{\rho}-2 \hat{\Lambda} \\
2 \frac{a^{\prime \prime}}{a}+\frac{n^{\prime \prime}}{n}-\frac{a^{\prime}}{a}\left(2 \frac{a^{\prime}}{a}+\frac{n^{\prime}}{n}\right)+\frac{1}{n^{2}}\left(\frac{\ddot{a}}{a}+\frac{\dot{a}}{a}\left(2 \frac{\dot{a}}{a}-\frac{\dot{n}}{n}\right)\right) & =0 .
\end{aligned}
$$


Of the above equations, (36) is remarkable in that it does not contain derivatives with respect to $t$. Thus, plugging in $n, a$ and $\rho$, which we have expressed in terms of functions of $t$ and functions of $y$, into (36), we obtain an algebraic equation involving functions of $t$. The details are again relegated to the Appendix. From this algebraic equation we see that $n$ and $a$ have the following factorizable form!:

$$
\begin{aligned}
& n(t, y)=n_{0}(t) f(y) \\
& a(t, y)=a_{0}(t) f(y) .
\end{aligned}
$$

Shifting to cosmic time, we arrive at the simple factorizable metric (9):

$$
\begin{aligned}
& n(t, y)=f(y) \\
& a(t, y)=a_{0}(t) f(y) .
\end{aligned}
$$

So, the presence of bulk ideal fluid leads (given our four assumptions) to the static configuration with a simple factorizable metric.

\subsection{Solution of the Einstein equation}

We have shown that when the bulk contains ideal fluid, our four assumptions lead to the factorizable metric (39). We now complete the solution of the Einstein equation with bulk and brane ideal fluids (of course, the brane ideal fluids reduce to cosmological constants, as we see from $(13))$.

With the factorizable metric (39), the equations (35) to (37) read

$$
\begin{aligned}
\hat{\rho} & =\frac{2}{3(w+1)}\left(\left(\frac{\dot{a}_{0}}{a_{0}}\right)^{2}-\frac{\ddot{a}_{0}}{a_{0}}\right) f^{-2} \\
\frac{f^{\prime \prime}}{f} & =\frac{w-1}{4} \hat{\rho}-\frac{\hat{\Lambda}}{2} \\
f^{\prime \prime} f-\left(f^{\prime}\right)^{2} & =-\frac{1}{3} \frac{\ddot{a}_{0}}{a_{0}}-\frac{2}{3}\left(\frac{\dot{a}_{0}}{a_{0}}\right)^{2} \equiv-\frac{\mathrm{C}}{9} .
\end{aligned}
$$

In the last equation we have introduced a constant C. The l.h.s. of (41) is independent of $t$, while from (40) we see that $\hat{\rho}$ depends on $t$. Thus, we obtain the result $w=1$ f. In other words, the only allowed equation of state for the bulk ideal fluid is

$$
p=\rho .
$$

\footnotetext{
${ }^{7}$ The special case $w=-\frac{1}{3}$ would yield a non-factorizable metric. However, this requires $\Lambda=0$, so the metric does not have exponential warping and is of no interest as regards the hierarchy problem.

${ }^{8}$ The possibility $\frac{\dot{a}_{0}^{2}}{a_{0}^{2}}-\frac{\ddot{a}_{0}}{a_{0}}=$ constant would reduce the bulk fluid to a cosmological constant.

${ }^{9}$ This result appeared in [16] in a different setting, valid only in the case $\dot{b} \neq 0$.
} 
This result does not depend on the choice $K=0$. We postpone discussion of the properties of an ideal fluid with this equation of state to section 3, and proceed to find the corresponding cosmological solutions.

With $w=1$, the equations (40) to (42) read

$$
\begin{aligned}
\hat{\rho} & =\frac{1}{3}\left(\left(\frac{\dot{a}_{0}}{a_{0}}\right)^{2}-\frac{\ddot{a}_{0}}{a_{0}}\right) f^{-2} \\
\frac{f^{\prime \prime}}{f} & =-\frac{\hat{\Lambda}}{2} \\
f^{\prime \prime} f-\left(f^{\prime}\right)^{2} & =-\frac{\mathrm{C}}{9} \\
\frac{\ddot{a}_{0}}{a_{0}}+2\left(\frac{\dot{a}_{0}}{a_{0}}\right)^{2} & =\frac{\mathrm{C}}{3} .
\end{aligned}
$$

For $K \neq 0$, the time dependence of $\hat{\rho}$ and the equation for $a_{0}$ would change, but the equations for the warp factor would remain unchanged. Note that the bulk cosmological constant contributes only to the warp factor, not to the cosmological expansion factor $a_{0}$. Interestingly, the bulk fluid accumulates at the brane where $f$ has its minimum value, in other words at the TeV brane, a result noted in [10].

Comparison with the FRW model. It is interesting to compare the equations (44) and (47) with the corresponding equations for the $K=0$ Friedmann-Robertson-Walker model. We take the same matter content for the FRW model: an ideal fluid with the equation of state $p_{(3)}=\rho_{(3)}$ and a cosmological constant $\Lambda_{(3)}$ (we use the subscript 3 as a reminder that there are only three spatial dimensions). The Einstein equation for the FRW model with this matter content can be written as

$$
\begin{aligned}
\hat{\rho}_{(3)} & =\frac{1}{3}\left(\left(\frac{\dot{a}}{a}\right)^{2}-\frac{\ddot{a}}{a}\right) \\
\frac{\ddot{a}}{a}+2\left(\frac{\dot{a}}{a}\right)^{2} & =3 \hat{\Lambda}_{(3)} .
\end{aligned}
$$

Comparison of (44) and (47) with (48) and (49) shows that $a_{0}$ in the RS case is exactly the same as $a$ in the FRW case, with $\mathrm{C}$ playing the role of a cosmological constant, $\mathrm{C}=9 \hat{\Lambda}_{(3)}$, and that $\hat{\rho}$ equals $\hat{\rho}_{(3)}$, save for the factor $f^{-2}$. These results hold also for $K \neq 0$. So, bulk ideal fluid in the RS model behaves exactly like ideal fluid in the FRW model, modulo the warp factor. However, we will shortly see that in the RS case only the sign of $\mathrm{C}$ has any physical meaning, in contrast to the FRW case, where the magnitude of $\hat{\Lambda}_{(3)}$ sets the timescale of the universe.

We analyze the cases $\mathrm{C}=0$ and $\mathrm{C} \neq 0$ separately. 
$\mathbf{C}=\mathbf{0}$. The equations (44) to (47) now read

$$
\begin{aligned}
\hat{\rho} & =\frac{1}{3}\left(\left(\frac{\dot{a}_{0}}{a_{0}}\right)^{2}-\frac{\ddot{a}_{0}}{a_{0}}\right) f^{-2} \\
\frac{f^{\prime \prime}}{f} & =-\frac{\hat{\Lambda}}{2} \\
f^{\prime \prime} f-\left(f^{\prime}\right)^{2} & =0 \\
\frac{\ddot{a}_{0}}{a_{0}}+2\left(\frac{\dot{a}_{0}}{a_{0}}\right)^{2} & =0 .
\end{aligned}
$$

The above equations have the following unique solution

$$
\begin{aligned}
a_{0}(t) & =\left(\frac{t}{\tau}\right)^{1 / 3} \\
f(y) & =f_{0} e^{ \pm y \sqrt{-\hat{\Lambda} / 2}} \\
\rho(t, y) & =\frac{1}{3 \kappa^{2}} \frac{1}{t^{2}} f(y)^{-2} .
\end{aligned}
$$

We have normalized by $a_{0}(0)=0$. The bulk cosmological constant has to be negative, $\hat{\Lambda}<0$.

The solution contains two free parameters, $\tau$ and $f_{0}$. A choice of the time parameter $\tau$ corresponds to choosing the unit of time, so that $\tau$ has no physical meaning, as in the FRW case. A choice of the warp parameter $f_{0}$ corresponds to choosing the origin of the $y$-coordinate. The metric and the energy density are invariant under the scaling $t \rightarrow \lambda t$, $x^{i} \rightarrow \lambda x^{i}, f_{0} \rightarrow \lambda^{-1} f_{0}$, so that $f_{0}$, or the placement of the origin, has no physical meaning.

The $\mathrm{C}=0$ solution contains no physical degrees of freedom other than the value of the bulk cosmological constant: the metric and the bulk energy density (and, as we will see, the brane cosmological constants) are fixed once the bulk cosmological constant is specified. This suggests that the solution is unstable, possibly collapsing to one of the $\mathrm{C} \neq 0$ solutions when perturbed.

$\mathbf{C} \neq \mathbf{0} . \quad$ In this case the equations $(44)$ to $(47)$ read

$$
\begin{aligned}
\hat{\rho} & =\frac{1}{3}\left(\left(\frac{\dot{a}_{0}}{a_{0}}\right)^{2}-\frac{\ddot{a}_{0}}{a_{0}}\right) f^{-2} \\
\frac{f^{\prime \prime}}{f} & =-\frac{\hat{\Lambda}}{2} \\
f^{\prime \prime} f-\left(f^{\prime}\right)^{2} & =-\frac{\mathrm{C}}{9} \\
\frac{\ddot{a}_{0}}{a_{0}}+2\left(\frac{\dot{a}_{0}}{a_{0}}\right)^{2} & =\frac{\mathrm{C}}{3} .
\end{aligned}
$$

\footnotetext{
${ }^{10}$ Apart from the trivial solution $\dot{a}_{0}=0$, which would lead to $\rho=0$ and the original RS model.
} 
The above equations have the solution!

$$
\begin{aligned}
a_{0}(t) & =a_{1} \begin{cases}\sin ^{1 / 3}(\sqrt{|\mathrm{C}|} t) & \mathrm{C}<0 \\
\sinh ^{1 / 3}(\sqrt{\mathrm{C}} t) & \mathrm{C}>0\end{cases} \\
f(y) & =f_{1} e^{y \sqrt{-\hat{\Lambda} / 2}}+\frac{\mathrm{C}}{18 \hat{\Lambda} f_{1}} e^{-y \sqrt{-\hat{\Lambda} / 2}} \\
\rho(t, y) & =\frac{|\mathrm{C}|}{3 \kappa^{2}} f(y)^{-2}\left\{\begin{array}{ll}
\sin ^{-2}(\sqrt{|\mathrm{C}|} t) & \mathrm{C}<0 \\
\sinh ^{-2}(\sqrt{\mathrm{C}} t) & \mathrm{C}>0
\end{array} .\right.
\end{aligned}
$$

We have again used the normalization $a_{0}(0)=0$. The equations for $f$ would allow a positive (zero) bulk cosmological constant, resulting in trigonometric functions (a linear function), but in order to solve the hierarchy problem we take $\hat{\Lambda}<0$.

As in the previous case, the parameter $a_{1}$ has no physical meaning, and can be set to unity, while $f_{1}$ corresponds to the choice of the origin of $y$. Rewriting $f_{1}$ in terms of a coordinate value $y_{0}$, we have

$$
\begin{aligned}
& a_{0}(t)= \begin{cases}\sin ^{1 / 3}(\sqrt{|\mathrm{C}|} t) & \mathrm{C}<0 \\
\sinh ^{1 / 3}(\sqrt{\mathrm{C}} t) & \mathrm{C}>0\end{cases} \\
& f(y)=\sqrt{\frac{2|\mathrm{C}|}{9|\hat{\Lambda}|}} \begin{cases}\cosh \left(\sqrt{|\hat{\Lambda}| / 2}\left(y-y_{0}\right)\right) & \mathrm{C}<0 \\
\sinh \left(\sqrt{|\hat{\Lambda}| / 2}\left(y-y_{0}\right)\right) & \mathrm{C}>0\end{cases} \\
& \rho(t, y)=\frac{|\Lambda|}{2}\left\{\begin{array}{ll}
\cosh ^{-2}\left(\sqrt{|\hat{\Lambda}| / 2}\left(y-y_{0}\right)\right) \sin ^{-2}(\sqrt{|\mathrm{C}|} t) & \mathrm{C}<0 \\
\sinh ^{-2}\left(\sqrt{|\hat{\Lambda}| / 2}\left(y-y_{0}\right)\right) \sinh ^{-2}(\sqrt{\mathrm{C}} t) & \mathrm{C}>0
\end{array} .\right.
\end{aligned}
$$

We see that the metric and the energy density are invariant under the scaling $t \rightarrow \lambda t$, $x^{i} \rightarrow \lambda x^{i}, \mathrm{C} \rightarrow \lambda^{-2} \mathrm{C}$. In other words, only the sign of $\mathrm{C}$ has any physical meaning, the magnitude is irrelevant. In particular, $\mathrm{C}$ does not introduce a new mass scale into the model.

Unlike in the case $\mathrm{C}=0$ (and the original $\mathrm{RS}$ proposal), the model is not invariant under translations of the $y$-coordinate, so that the choice of origin of $y$ does have a physical meaning. The translational invariance is broken by the $y$-dependence in the bulk matter: $y_{0}$ is not a free parameter but is set by the bulk energy density, as we see from (66). There are two physical degrees of freedom: the value of the bulk cosmological constant and the bulk energy density. (In addition, there are of course the brane cosmological constants, which, as we will see shortly, are also free parameters.)

In the case $\mathrm{C}>0$ the function $f$ has a zero at $y=y_{0}$. The bulk energy density and the scalar curvature diverge when $f=0$, so the singularity at $y=y_{0}$ is physical, not an

\footnotetext{
${ }^{11}$ The equation for $a_{0}$ allows de Sitter and anti-de Sitter solutions when $\mathrm{C}>0$, but these would again give $\rho=0$. These solutions were first presented in [17, 29].
} 
artifact of the coordinate system we have chosen. In what follows, we simply avoid the singularity by constraining the value $y_{0}$ not to lie between the brane positions in the case $\mathrm{C}>0$.

\subsection{Branes and fine-tuning}

Having solved the Einstein equation in the bulk, we now turn to the branes, which, as noted earlier, provide boundary conditions.

Brane cosmological constants. The factorizable metric implies that the branes contain only cosmological constants. We switch to the notation $\rho_{m}=-p_{m}=: \Lambda_{m}$. Inserting the brane contribution to the stress tensor from (23) into the Einstein equation (41), we get

$$
\left.\frac{\left[f^{\prime}\right]}{f}\right|_{y=y_{m}}=-\hat{\Lambda}_{m}
$$

where $y_{1}, y_{2}\left(y_{2}>y_{1}\right)$ are the orbifold fixed points, where the branes are located $\square$. As before, the notation $\left[f^{\prime}\right]$ refers to the discontinuity of $f^{\prime}$. Writing the discontinuity in terms of the continuous part $f_{c}^{\prime}$, we have

$$
\begin{aligned}
\left.2 \frac{f_{c}^{\prime}}{f}\right|_{y=y_{1}} & =-\hat{\Lambda}_{1} \\
-\left.2 \frac{f_{c}^{\prime}}{f}\right|_{y=y_{2}} & =-\hat{\Lambda}_{2} .
\end{aligned}
$$

For $\mathrm{C}=0$ (and the original RS proposal) we have $f(y)=f_{0} e^{-|y| \sqrt{|\hat{\Lambda}| / 2}}$. Then (68) gives the result $\hat{\Lambda}_{1}=-\hat{\Lambda}_{2}=\sqrt{2|\hat{\Lambda}|}$, an unfortunate fine-tuning. However, for $\mathrm{C}<0$ we have instead

$$
\begin{aligned}
& \hat{\Lambda}_{1}=-\sqrt{2|\hat{\Lambda}|} \tanh \left(\sqrt{|\hat{\Lambda}| / 2}\left(y_{1}-y_{0}\right)\right) \\
& \hat{\Lambda}_{2}=\sqrt{2|\hat{\Lambda}|} \tanh \left(\sqrt{|\hat{\Lambda}| / 2}\left(y_{2}-y_{0}\right)\right)
\end{aligned}
$$

for $\mathrm{C}>0$ the hyperbolic tangent is replaced by a hyperbolic cotangent ${ }^{\mathrm{T}} \mathrm{T}$. The above equations for the case $\mathrm{C}<0$ were found in [7] in a slightly different setting. The values of hyperbolic tangent lie in the interval from -1 to +1 (excluding \pm 1 ), and the values of hyperbolic cotangent stretch from minus infinity to plus infinity, excluding the interval

\footnotetext{
${ }^{12}$ In the case $\mathrm{C}>0$ we demand $y_{0}<y_{1}$ or $y_{0}>y_{2}$ to avoid having a singularity.

${ }^{13}$ Note that either set of equations fixes $y_{2}-y_{1}$, the size of the fifth dimension.
} 
from -1 to +1 . Thus, the ratio of the brane cosmological constants to the square-root of the bulk cosmological constant determines the value of $\mathrm{C}$ :

$$
\begin{array}{cc}
\mathrm{C}<0 & \text { for } \frac{\left|\hat{\Lambda}_{m}\right|}{\sqrt{2|\hat{\Lambda}|}}<1 \\
\mathrm{C}=0 & \text { for } \frac{\left|\hat{\Lambda}_{m}\right|}{\sqrt{2|\hat{\Lambda}|}}=1 \\
\mathrm{C}>0 & \text { for } \frac{\left|\hat{\Lambda}_{m}\right|}{\sqrt{2|\hat{\Lambda}|}}>1 .
\end{array}
$$

In the present model, the Einstein equation implies no fine-tuning of parameters, unlike in the original RS proposal. For a given value of the bulk cosmological constant $\hat{\Lambda}$, the absolute values of both brane cosmological constants have to be smaller than, bigger than or equal to $\sqrt{2|\hat{\Lambda}|}$, but are otherwise unrestricted by the Einstein equation. With two equations to satisfy and two constants, $y_{1}-y_{0}$ and $y_{2}-y_{0}$, at our disposal, no fine-tuning is needed to obtain a solution to (68).

The signs of the brane cosmological constants are opposite for $\mathrm{C}=0$ and $\mathrm{C}>0$. This can also be the case for $\mathrm{C}<0$. However, for $\mathrm{C}<0$ it is also possible for both brane cosmological constants to be positive: this requires the value of $y_{0}$ to lie between the brane positions, $y_{1}<y_{0}<y_{2}$. Furthermore, we have the interesting possibility of a zero cosmological constant on one brane and a positive cosmological constant on the other: this requires one of the branes to be placed at the point where $f$ has its minimum, $y_{0}=y_{1}$ or $y_{0}=y_{2}$. (It is not possible to have two branes with non-positive cosmological constants.) Thus, there is no need for negative energy densities on the branes, essentially because the warp factor is not a monotonical function, but has a minimum. This was first noticed in [17] and further emphasized in [7]. Of course, the bulk cosmological constant still has to be negative to obtain an exponential warp factor.

The hierarchy problem. The solution to the hierarchy problem introduces a new equation, namely

$$
\frac{f\left(y_{1}\right)}{f\left(y_{2}\right)} \sim 10^{16}
$$

For $\mathrm{C}<0$, the above equation reads

$$
\frac{\cosh \left(\sqrt{|\hat{\Lambda}| / 2}\left(y_{1}-y_{0}\right)\right)}{\cosh \left(\sqrt{|\hat{\Lambda}| / 2}\left(y_{2}-y_{0}\right)\right)} \sim 10^{16} ;
$$


for $\mathrm{C}>0$ the hyperbolic cosine is replaced by a hyperbolic sine. Combining (72) with (69), we can write the hierarchy condition (71) as the following equation, valid for all values of $\mathrm{C}$ :

$$
\frac{\hat{\Lambda}_{1}^{2}}{2|\hat{\Lambda}|}-1 \sim 10^{32}\left(\frac{\hat{\Lambda}_{2}^{2}}{2|\hat{\Lambda}|}-1\right) .
$$

We see that the only way to avoid introducing unnaturally large numbers is to fine-tune the cosmological constants, $\hat{\Lambda}_{1}^{2}=\hat{\Lambda}_{2}^{2}=2|\hat{\Lambda}|$, corresponding to $\mathrm{C}=0$.

The above result is not dependent on the presence of bulk ideal fluid: if we put $\rho=0$, our solutions disappear, but we get new solutions with the same problems. For $\mathrm{C}=0$ we get a constant $a_{0}$ (the original $\mathrm{RS}$ solution) and for $\mathrm{C}>0$ we get de Sitter and anti-de Sitter solutions, found in [17, 29]; the case $\mathrm{C}<0$ becomes disallowed. The warp factor is unchanged by the absence of bulk ideal fluid (except that $y_{0}$ becomes a free parameter), so the condition (73) is also unchanged. This means that the fine-tuning problem is not inherent to the Einstein equation. Specifically, it is not due to the condition $\dot{b}=0$, as sometimes claimed [3]. It is rather a rephrasing of the hierarchy problem. In this sense the RS model does not provide quite a satisfactory solution to the hierarchy problem.

\section{Discussion}

As is well known, the RS solution is quite precarious; bulk and brane sources for gravity must be chosen very carefully for the RS solution to emerge from the Einstein equation. However, it turns out that in two-brane systems one easily end up with severe constraints for the sources; this fact is not apparent from the literature. We hope that our results help to identify the potential problem sources in trying introduce realistic matter into the bulk or branes, while preserving the main features of the RS scenario: the hierarchy generation and the localization of gravity. The need to include generic brane matter in particular creates problems in trying to interpret our universe as a brane world.

In Section 2 we found that under minimal assumptions on the RS model, a stabilized radion and zero thickness branes, one cannot introduce realistic brane matter 4 in static two-brane configurations. The only allowed equation of state, other than that of a cosmological constant, corresponds to a fluid of two dimensional domain walls moving at the speed of light. Even that requires giving up factorizability of the bulk metric. Further relaxation of the constraints for brane matter requires a time-dependent and non-factorized warp factor, and most likely a slow stabilization of the radion as the branes settle to the final fixed proper distance. This idea is supported by the result of Section 3, which shows

\footnotetext{
${ }^{14}$ In this context, we mean homogeneous and isotropic matter with equations of state corresponding to a combination of dust and radiation.
} 
that when the bulk is filled with ideal fluid and the radion is completely stabilized, the warp factor is necessarily factorizable. The constraint for the equation of state of the bulk ideal fluid also points in this direction.

The dynamical stabilization of the radion degree of freedom can be expected to be highly nontrivial. Nevertheless, unless for some reason the universe started out with all the degrees of freedom in the ground state, some dynamics should be expected. In the context of cosmology, a natural initial condition for the radion could be any value compatible with the uncertainty principle, as in chaotic inflation. The radion potential energy must then be released in some way. It is conceivable that the extra radion energy is dissipated into purely gravitational degrees of freedom, but there is no guarantee that a RS-type solution would result; this remains to be studied. Dissipation into fields living on the brane has been studied in [3]. The third possibility, radion decaying into bulk degrees of freedom, assumed in this paper, is possible but highly constrained, as we have shown. The only admissible equation of state for ideal fluid in the bulk was found to be $p=\rho$, representing the so-called stiff ideal fluid. Here "stiff" reflects the fact that the velocity of sound in the fluid is equal to the velocity of light. Concretely, such a fluid corresponds to a classical free massless, coherent scalar field (not to be confused with massless radiation), something that was considered in a static setting in [7]. Further, the massless bulk scalar field should not be confused with the massive Goldberger-Wise bulk scalar field. Whether such a field could be coupled to the radion is beyond the scope of the present study.

The no-go flavor of our results is meant to clarify what are the most promising directions for modifying the two-brane models so that other equations of state for the brane or bulk ideal fluids can be allowed. In the case of the bulk, a natural guess would be to start with a different Ansatz for the bulk geometry. This corresponds to a different assumption about the spacetime symmetries. For example, if the bulk geometry would correspond to an AdS black hole, one might expect the equation of state for massless radiation to become allowed.

\section{Acknowledgements}

KE was partly supported by the Academy of Finland under the contract 101-35224. We would like to thank W. Goldberger, C. Montonen, L. Randall, and M. Wise for some helpful discussions as we were getting into this subject. We also thank J. Cline, A. Karch, D. Langlois, T. Shiromizu, and their collaborators for correspondence on the topic of constraints for brane matter. 


\section{Appendix: Bulk Ideal Fluid and Factorization}

We present here details of the calculation of section 3.2 which shows that the presence of bulk ideal fluid leads to a factorizable metric. The starting point was the equations (32):

$$
\begin{aligned}
\rho(t, y) & =\rho_{0} B(t)^{\frac{1+w}{w}} n(t, y)^{-\frac{1+w}{w}} \\
n(t, y) & =B(t) C(y) a(t, y)^{3 w} \\
\dot{a}(t, y) & =A(t) B(t) C(y) a(t, y)^{3 w} .
\end{aligned}
$$

Integrating the equation for $a$, we obtain the following expressions for $n, a$ and $\rho$ :

$$
\begin{aligned}
& w=\frac{1}{3} \quad\left\{\begin{array}{l}
\rho(t, y)=\rho_{0} C(y)^{-4} e^{-4(C(y) E(t)+D(y))} \\
n(t, y)=B(t) C(y) e^{C(y) E(t)+D(y)} \\
a(t, y)=e^{C(y) E(t)+D(y)}
\end{array}\right. \\
& w \neq \frac{1}{3}\left\{\begin{array}{l}
\rho(t, y)=\rho_{0} C(y)^{-\frac{1+w}{w}}(C(y) E(t)+D(y))^{-3 \frac{1+w}{1-3 w}} \\
n(t, y)=B(t) C(y)(C(y) E(t)+D(y))^{\frac{3 w}{1-3 w}} \\
a(t, y)=(C(y) E(t)+D(y))^{\frac{1}{1-3 w}}
\end{array}\right.
\end{aligned}
$$

In the above expression, $D(y)$ is a new unknown function, and $E(t)$ is proportional to the integral of $A(t) B(t)$. We have now separated the $t$ - and $y$-dependence of the metric. The linear combination of components of the Einstein equation which does not involve derivatives with respect to $t$ is $(36)$ :

$$
3 \frac{a^{\prime \prime}}{a}+\frac{n^{\prime \prime}}{n}=(w-1) \hat{\rho}-2 \hat{\Lambda}
$$

Now we simply insert (75) into (76). Let us consider the cases $w=\frac{1}{3}$ and $w \neq \frac{1}{3}$ separately.

$\mathbf{w}=\frac{\mathbf{1}}{\mathbf{3}} . \quad$ In this case (76) reduces to

$$
\begin{array}{r}
4 C^{\prime 2} E(t)^{2}+\left(4 D^{\prime \prime}+8 C^{\prime} D^{\prime}+2 \frac{C^{\prime 2}}{C}\right) E(t)+\left(4 D^{\prime \prime}+4 D^{\prime 2}+\frac{A^{\prime \prime}}{A}+2 \frac{C^{\prime}}{C} D^{\prime}\right) \\
=(w-1) \hat{\rho}_{0} C^{-4} e^{-4(C E(t)+D)}-2 \hat{\Lambda} .
\end{array}
$$

It is impossible for the above equation to be satisfied at all times $t$ unless $\dot{E}=0$ or $C=0$, both of which correspond to $\dot{a}=0$, and thus static cosmology. 
$\mathbf{w} \neq \frac{1}{3} . \quad$ Now $(76)$ can be written as

$$
\begin{array}{r}
\frac{6 w(1+3 w)}{(1-3 w)^{2}} D^{2}\left(\frac{D^{\prime}}{D}-\frac{C^{\prime}}{C}\right)^{2}(C E(t)+D)^{-2} \\
+\frac{3(1+w)}{1-3 w} D\left(\frac{D^{\prime \prime}}{D}-\frac{C^{\prime \prime}}{C}+\frac{6 w}{1-3 w} \frac{C^{\prime}}{C}\left(\frac{D^{\prime}}{D}-\frac{C^{\prime}}{C}\right)\right)(C E(t)+D)^{-1} \\
+\frac{4}{1-3 w}\left(\frac{C^{\prime \prime}}{C}+\frac{3 w}{1-3 w} \frac{C^{\prime 2}}{C^{2}}\right) \\
=(w-1) \hat{\rho}_{0} C^{-\frac{1+w}{w}}(C E(t)+D)^{-3 \frac{1+w}{1-3 w}}-2 \hat{\Lambda} .
\end{array}
$$

The above equation is less transparent than (77). Unless $w=-\frac{1}{3}$, there are three powers of $C E+D$ on the l.h.s., so by equating the coefficients of the powers of $C E+D$ we obtain three equations. These equations can only be satisfied if $D=0$ (or $D \propto C$, which amounts to the same thing by a redefinition of $C$ ). From (75) we see that this corresponds to a factorizable metric. This solution also requires $w=1$, though for reasons of clarity we preferred to present this result at a later stage in section 3 .

As for the special case $w=-\frac{1}{3}$, the above equation then does have solutions with a non-factorizable metric. However, a substitution to the full Einstein equation (35)-(37) shows that this requires $\Lambda=0$, and as a result the metric does not have an exponential warp factor and is of no interest as far as the hierarchy problem is concerned.

\section{References}

[1] Lisa Randall and Raman Sundrum. A large mass hierarchy from a small extra dimension. Phys. Rev. Lett., 83:3370, 1999. hep-ph/9905221.

[2] Lisa Randall and Raman Sundrum. An alternative to compactification. Phys. Rev. Lett., 83:4690, 1999. hep-th/9906064.

[3] Csaba Csáki, Michael Graesser, Lisa Randall and John Terning. Cosmology of brane models with radion stabilization hep-ph/9911406.

[4] Walter D. Goldberger and Mark B. Wise. Modulus stabilization with bulk fields. Phys. Rev. Lett., 83:4922, 1999. hep-ph/9907447. Bulk Fields in the Randall-Sundrum Compactification Scenario. hep-ph/9907218.

[5] James M. Cline and Hassan Firouzjahi. Brane-world cosmology of modulus stabilization with a bulk scalar field. hep-ph/0005235. 
[6] O. DeWolfe, D.Z. Freedman, S.S. Gubser and A. Karch. Modeling the fifth dimension with scalars and gravity. hep-th/9909134.

[7] Panagiota Kanti, Keith A. Olive and Maxim Pospelov. Static solutions for brane models with a bulk scalar field. hep-ph/0002229.

[8] Markus A. Luty and Raman Sundrum. Radius stabilization and anomaly-mediated supersymmetry breaking. hep-th/9910202.

[9] Paul J. Steinhardt. General considerations of the cosmological constant and the stabilization of moduli in the brane-world picture. hep-ph/9907080.

[10] Jihn E. Kim and Bumsok Kyae. Exact cosmological solution and modulus stabilization in the Randall-Sundrum model with bulk matter. hep-th/0005139.

[11] Panagiota Kanti, Ian. I. Kogan, Keith A. Olive and Maxim Pospelov. Cosmological 3-brane solutions. hep-ph/9909481.

[12] Panagiota Kanti, Ian I. Kogan, Keith A. Olive and Maxim Pospelov. Single-brane cosmological solutions with a stable compact extra dimension Phys. Rev., D61:106004, 2000. hep-ph/9912266.

[13] Panagiota Kanti, Keith A. Olive and Maxim Pospelov. Solving the hierarchy problem in two-brane cosmological models. hep-ph/0005146.

[14] Hang Bae Kim. Cosmology of Randall-Sundrum models with an extra dimension stabilized by balancing bulk matter. hep-th/0001209.

[15] R.N. Mohapatra, A. Pérez-Lorenzana and C.S. de S. Pires. Cosmology of brane-bulk models in five dimensions. hep-ph/0003328.

[16] Conall Kennedy and Emil M. Prodanov. Standard cosmology from sigma-model. hep-th/0003299.

[17] Nemanja Kaloper. Bent domain walls as braneworlds. hep-th/9905210.

[18] André Lukas, Burt A. Ovrut and Daniel Waldram. Boundary inflation. hepth/9902071.

[19] Martin Gremm. Thick domain walls and singular spaces. hep-ph/0002040.

[20] Nima Arkani-Hamed, Savas Dimopoulos, Nemanja Kaloper and Raman Sundrum. A small cosmological constant from a large extra dimension. hep-th/0001197. 
[21] Julien Lesgourges, Sergio Pastor, Marco Peloso and Lorenzo Sorbo. Cosmology of the Randall-Sundrum model after dilaton stabilization. hep-ph/0004086.

[22] Pierre Binétruy, Cédric Deffayet and David Langlois. Non-conventional cosmology from a brane-universe. hep-ph/9905012.

[23] T. Shiromizu, K. Maeda and M. Sasaki. The Einstein equations on the 3-brane world. Phys. Rev., D62:024012, 2000. gr-qc/9910076.

[24] Pierre Binétruy, Cédric Deffayet, Ulrich Ellwanger and David Langlois. Brane cosmological evolution in a bulk with cosmological constant. hep-th/9910219.

[25] P. Kraus. Dynamics of anti-de Sitter domain walls- JHEP 9912, 011, 1999. hepth/9910149.

[26] D. Ida. Brane-world cosmology. gr-qc/9912002.

[27] S. Mukohyama, T. Shiromizu and K. Maeda. Global structure of exact cosmological solutions in the brane world. Phys. Rev., D62:024028, 2000. hep-th/9912287.

[28] Benjamin Grinstein, Detlef R. Nolte and Witold Skiba. Adding matter to Poincare invariant branes. hep-th/0005001.

[29] Takeshi Nihei. Inflation in the five-dimensional universe with an orbifold extra dimension. hep-ph/9905487. 